\title{
Research on Vision-based Position Measurement System for Rocket Propellant Loading
}

\author{
Tao Han ${ }^{1, \mathrm{a}}$ and Cong Dacheng ${ }^{1}$ \\ ${ }^{1}$ State Key Laboratory of Robotics and System, Harbin Institute of Technology, Harbin, China
}

\begin{abstract}
In the process of rocket propellant loading, the propellant's own gravity and wind pendulum may cause the connector to exert a large force on the rocket body, which may lead to connector disconnection and fuel leakage, or even damage to the rocket body. To improve the reliability and safety of rocket propellant loading, a position measurement system based on dynamic orientation detection alignment technology is proposed to achieve tracking of the rocket refueling port. In this paper, the two-dimensional position of the target in the camera plane is quickly extracted by identifying the direction of the target image and the size of the marker in the image, while the distance between the target and the camera is accurately obtained by a laser rangefinder. With this measurement solution, the deviation of the connector from the propellant filler port at the current moment can be modified in real time, thus reducing the forces on the rocket and increasing its launch reliability.
\end{abstract}

\section{Introduction}

When the carrier rocket is in the propellant loading state after arriving at the launch position, it makes small irregular swings under the influence of the wind pendulum $[1,2]$. This literature analyses the effect of the motion of the rocket body on the connector, which is driven by the rocket body to make small irregular swings. Once the propellant loading process begins, the height of the rocket body slowly sinks as the amount of rocket propellant refuelled increases. As the rocket body sways and sinks, the connector mounted on the pendulum of the launch tower moves at the same time, and the connector is solidly connected to the loading port valve on the rocket body, which causes a large internal stress on the rocket body and affects the reliability and tightness of the connection [3]. Therefore, it is necessary to investigate an automatic docking device that can follow the movement of the rocket body after the connector is first connected, and can automatically dock the connector interface and valve after the connector is accidentally disconnected.

There are currently two different approaches to rocket fueling in the world: the Russian "rack-mounted" approach and the American "rocket-mounted" approach. In the literature [4], it is mentioned that the Russian technology uses tactile detection as the main means of measuring deviation, and although the structure is simple and reliable and has a secondary docking function, its core is a rigid assembly. Inevitably, this has the disadvantage of high assembly requirements and large equipment size. The National Aeronautics and Space Administration (NASA) has mentioned in the literature [5] the development of a remote intelligent umbilical docking system that can be used for carrier rockets [6]. As shown in Figure 1, this automatic docking system allows for the connection, separation and re-docking of the rocket body at any given moment [7]. During the connector docking process, the camera installed in the docking system detect the displacement deviation caused by the swing of the rocket body, and the control terminal of the automatic docking system receives the spatial position information from the sensors.

In this paper, a vision measurement system is designed for automatic docking deviations. The position of a specific mark (artificial target) is detected in real time by an optical camera, while the distance between the connector and the rocket is measured by a laser distance sensor to obtain the Cartesian coordinates of the rocket's filler port [8]. The actuators are controlled to adjust the position of the connector so that the connector is precisely aligned with the docking port on the rocket body. Once docking and locking is complete, the connector swings with the rocket body. The use of the follower technology greatly reduces the force of the connector and its piping on the rocket body interface, which is beneficial to the structure of the rocket body, and allows direct docking and uncoupling from the rocket body at any time, greatly reducing the docking and uncoupling time, contributing to rapid rocket launch and improving safety and reliability.

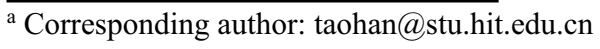




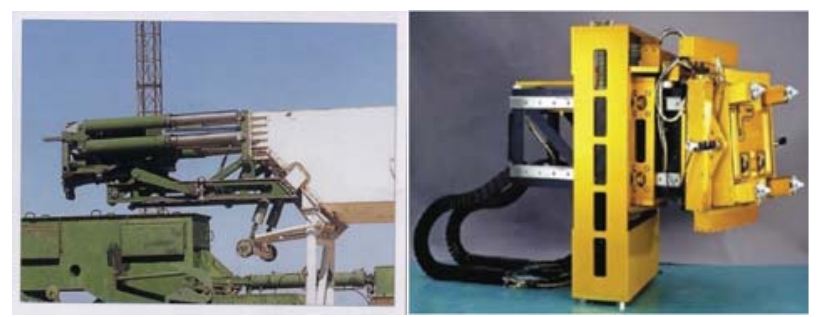

Fig. 1. Hydraulic manipulator and target object

\section{System description}

\subsection{System components}

The entire experimental system consists of a hydraulic parallel manipulator and a motion simulator of the target object. In this section, the various parts of the system are described in detail. After that, the working principle of the vision system employed in the article is introduced, and the kinematics and dynamics modeling of the platform are carried out.

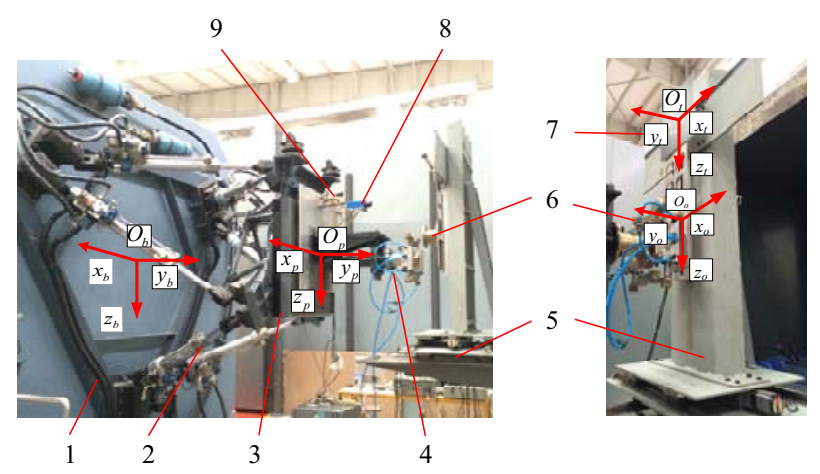

Fig. 2. Hydraulic manipulator and target object 1.Platform Basement 2.HPM 3.Force Sensor 4. End-effector 5.Slideway 6. Fuel Filler Port 7.Planar pattern 8.Laser rangefinder 9.CCD camera

The composition of the entire experimental system is shown in Figure 2. Located in the middle of the figure is a horizontal hydraulic parallel manipulator, the lower platform is fixed on the base, and the upper platform can freely move in the workplace to capture the object equipped with a docking device. The manipulator is driven by 6 hydraulic actuators and each actuator is connected with upper and lower platform by Hooke Joint. Wherein, each hydraulic actuator is composed of an asymmetric servo valve and an asymmetric hydraulic cylinder. A plane six-degree-of-freedom force sensor is arranged between the upper platform and the docking device to measure the force and torque that interact with the environment.

\subsection{Vision system}

The method proposed in this work to localize the parallel manipulator and estimate the target's status are based on information fusion of a monocular camera and laser rangefinder.
As shown in the Fig. 3, firstly the image acquisition module collects the target video image input by the camera in real time and the target detection module quickly confirms the distance of the target by pattern matching; then the target center detection and orientation modules calculate the position and orientation of the target by image segmentation and target tracking according to the distance of the target; the target center estimation module calculates the target center's position by bundle adjustment according to the location of the target element; the target orientation estimation module will calculate the main direction of the target according to the position of the direction element and outputs the rotation angle [9].

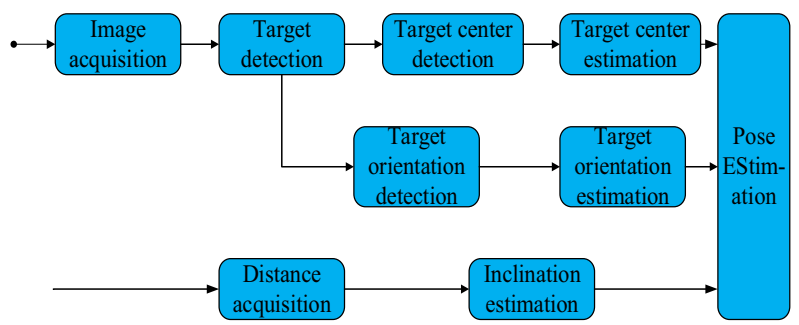

Fig. 3. Vision system work process

\section{Vision sensor modelling}

\subsection{Imaging system}

The position recognition of the target depends on the accurate extraction of the target and directional elements in the target image, and it must be ensured that the camera can image the target and directional elements clearly throughout the working travel range [10].

At the furthest end of the camera's working stroke, $\mathrm{D}=1.2 \mathrm{~m}$, the physical space covered by the image is at least $300 \mathrm{~mm} \times 200 \mathrm{~mm}$ according to the technical specifications, so that the target image does not overflow into the field of view, and therefore the spatial extent is $\mathrm{L}=1 \mathrm{~m}$. At the same focal length, the horizontal direction will cover a larger spatial area than the vertical direction, so only the vertical direction is considered. Only the vertical direction is considered. Let the length of the spatial extent correspond to an image length of $1=2.88$ $\mathrm{mm}$, and calculate the focal length $f$ of the lens according to the geometrical optics of thin lens imaging.

$$
f=D \cdot \frac{l}{L}=1200 \mathrm{~mm} \times \frac{2.88 \mathrm{~mm}}{1000 \mathrm{~mm}}=3.456 \mathrm{~mm}
$$

\subsection{Target recognition}

To identify the position and pose of the target, the target must first be accurately detected, the target and direction elements in the target must be accurately extracted, and then the key parameters must be estimated through robust statistics. Figure 4 shows the results of target image analysis.

In Figure 4, (a) is the original target image; ( b) is the binary image; (c) is the target detection result; (d) is the target detection result; (e) is the bullseye estimation 
result, and the target position is marked by a green cross; (f) is the estimated result of the main direction of the target .

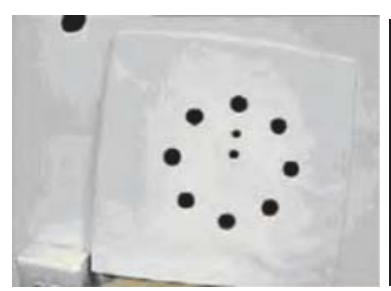

(a) The original image

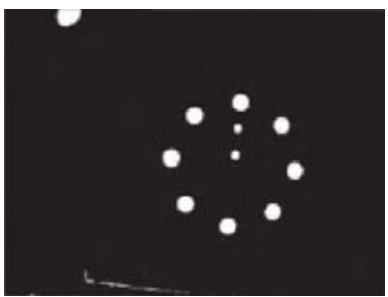

(c)Binary image

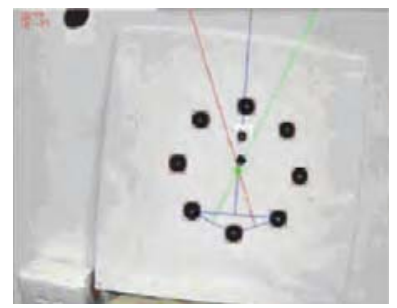

(e) Target center estimation

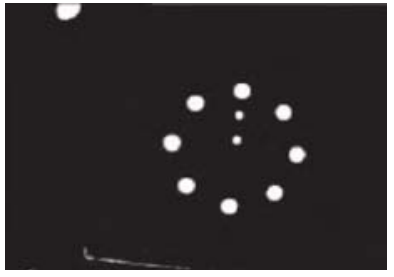

(b) Binary image

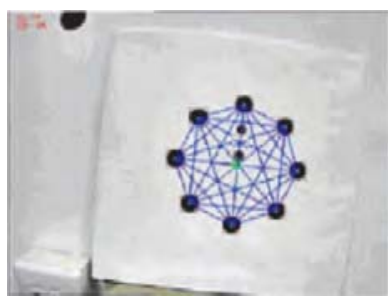

(d)Target center detection

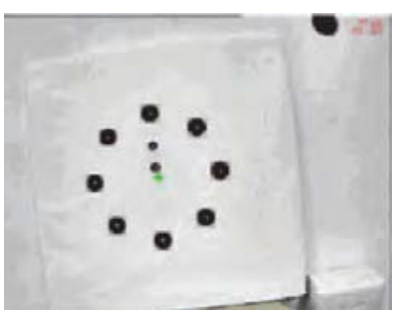

(f) Target orientation estimation Fig. 4. Target recognition modelling

\subsection{Pose recognition}

This scheme uses 3 laser ranging sensors to realize the angle measurement installation relationship and position, as shown in Fig. 2. The three laser ranging sensors measure the distance between the camera and the target separately, estimate the inclination along the line of sight of the camera by analyzing the distance difference, and output the pitch angle $\boldsymbol{\beta}$ and the azimuth angle $\boldsymbol{\alpha}$. The calculation principles of the two are the same [11].

To estimate the azimuth angle, for example, two laser distance sensors 1 and 3 are paired to measure the distance in parallel at a constant distance D31 and obtain the distance difference $\boldsymbol{\Delta} \boldsymbol{d}$.

A complete description of the relative position of the camera and the target requires six parameters, namely the position of the camera's optical centre on the three axes of the target coordinate system $(\boldsymbol{x}, \boldsymbol{y}, \boldsymbol{z})$ and the inclination of the camera's optical axis around the three axes $(\boldsymbol{\alpha}, \boldsymbol{\beta}, \boldsymbol{\gamma})$. Based on the distance $\boldsymbol{Z}_{d}$ from the camera's optical center to the intersection of the target surfaces, the coordinates of the camera's optical center in the target coordinate system $\left(\boldsymbol{x}_{\mathrm{c}}, \boldsymbol{y}_{\mathrm{c}}, \boldsymbol{z}_{\mathrm{c}}\right)$ can be obtained by a simple calculation.

$$
\begin{gathered}
x_{c}=-z_{d} \cdot \sin \alpha \cdot \cos \gamma+z_{d} \cdot \sin \beta \cdot \sin \gamma \\
y_{c}=-z_{d} \cdot \sin \alpha \cdot \sin \gamma+z_{d} \cdot \sin \beta \cdot \cos \gamma \\
z_{c}=\mathrm{z}_{d} \cdot \cos \alpha \cdot \cos \beta
\end{gathered}
$$

By correcting for the deviation of the bullseye position $\left(\boldsymbol{x}_{d}, \boldsymbol{y}_{d}\right)$, the correct coordinates of the camera, the correct coordinates of the camera in the target coordinate system.

$$
\begin{gathered}
x=x_{d} \frac{z_{c}}{f}-x_{c} \\
y=y_{d} \frac{z_{c}}{f}-y_{c} \\
z=z_{c}
\end{gathered}
$$

\section{Experimental result}

The overall experiment system includes two subsystems as shown in Fig. 5. One part consists of the hydraulic parallel manipulator and the system controller which includes a hydraulic source manager and motion controller. The other part of the system consists of a docking device and target movement simulator which includes simulator manager PC2 and the target motion simulator. The target motion simulator is used to move the target object along $x-y-z$ three orthogonal directions of Cartesian coordinate in order to simulate the target's realistic motion in the environment, and the simulator manager PC2 is employed to control the docking device to realize tracking, locking, unlocking and other tasks with the pose information acquired from the vision sensor.

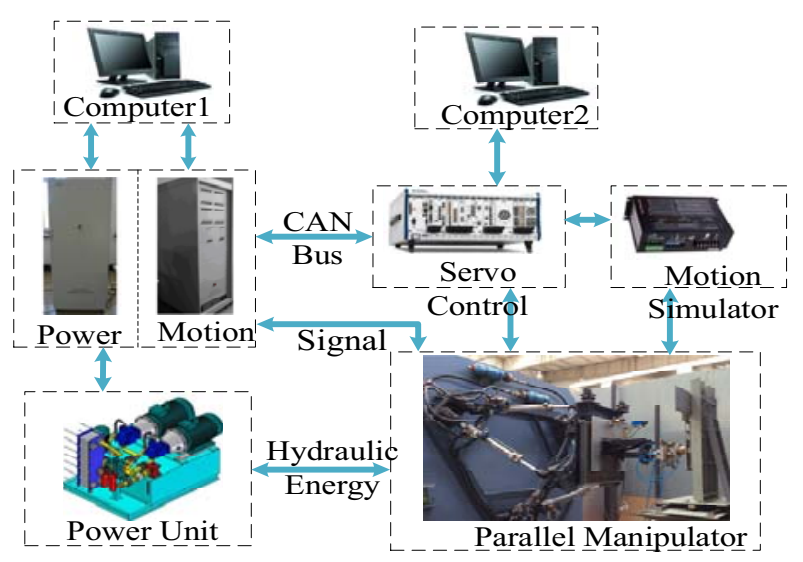

Fig. 5. Experimental system structure

The controllers of the two subsystems are both based on NI PXI-8196 embedded controller which communicate with $\mathrm{CAN}$ bus. And the vision sensor system is composed of TFN-0130 laser rangefinder with $1 \mathrm{~mm}$ measuring accuracy and a SONY Super HAD CCD camera with $658 \times 492$ image resolution. To measure the interact force during the whole contact process, we employ 6-dof force plate named BERTEC FP9090 which has a full scale of $2000 \mathrm{~N}$ force in the $x$ direction, $10000 \mathrm{~N}$ force in $y$ direction and $z$ direction, $4500 \mathrm{Nm}$ torque around $x$ direction.

The test platform for the measurement system is shown in Fig. 5, where the parallel mechanism allows for six degrees of freedom of movement and the target simulation tooling is mounted on a 3-do sliding table. The measurement system recognises the target image 
and distance information to automatically align the connector with the rocket fuel filler port.

As the aim of the experiment is to check the accuracy of the measuring system, the parallel mechanism is adjusted so that the mounting base of the measuring system on the parallel mechanism reduces the measurement errors caused by the unparallel mounting surfaces. The spatial position of the parallel mechanism is kept fixed, the slide is driven and the data is recorded. The relative displacement measurement error curves for the three axes are shown in Fig. 6, with the vertical coordinates showing the relative displacement changes in the three directions. As can be seen, the measurement accuracy of the system meets the technical requirements and can be measured to an accuracy of $1 \mathrm{~mm}$.

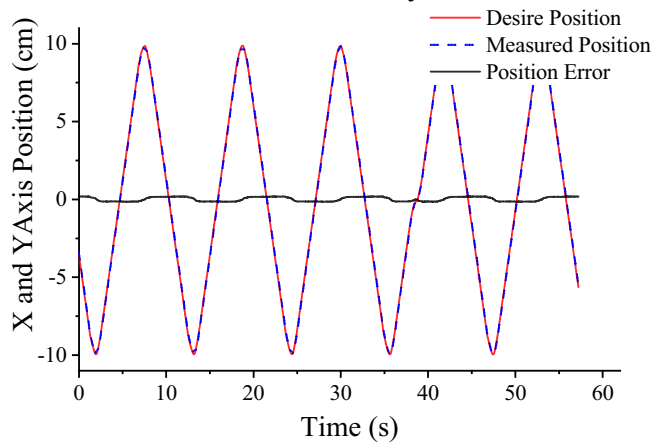

(a)

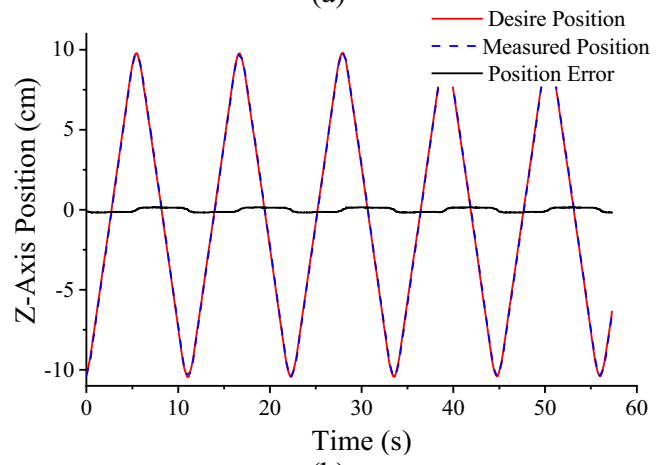

(b)

Fig. 6. Position measurement experiment

\section{Conclusions}

In order to realize the automatic docking and separation of the docking connector and the rocket fuel port before the rocket lifts off, this paper proposes a vision-based automatic docking deviation measurement system. It is verified by experiments that the scheme is feasible. By indirectly obtaining the position and attitude of the rocket fuel filler port through the attitude recognition of the artificial target, the automatic docking and following of the connector and port can be achieved, which can avoid excessive stress on the rocket fuel filler port due to the inability of the connector to follow, improve the reliability of the connector seal, and help improve the launch efficiency and safety.

\section{References}

1. X Dun, J Yuan, L Chen, ROBIO 2006, 485-490 (2006)
2. M Delrobaei, KA McIsaac, CCECE 2008, 001025001030 (2008)

3. AM Gosselin, Space Visions Congress, (2007)

4. E Artono, S Salman, Jurnal Teknologi Dirgantara, 16(2), 101-110 (2019)

5. Z Chenyang, J Hongzhou, Energies, 14(4), 941, 2021

6. G Prasad, E Tajudeen, Modeling, Simulation, and Verification of Space-based Systems II, 5799, 20-27 (2005)

7. VA Prabhu, A Tiwari, W Hutabarat, J Thrower, C Turner, Procedia CIRP, 25, 161-168 (2014)

8. ZY Zhang, J Zhang, DY Zhu, Acta Aeronautica et Astronautica Sinica, 28(4), 943-947 (2007)

9. G Xuehai, X Kejun, Z Han, L Xiaoli, Chinese journal of scientific instrument, 28(8), 1479 (2007)

10. KO Arras, $\mathrm{N}$ Tomatis, European Workshop on Advanced Mobile Robots 1999,177-185 (1999)

11. H Tao, ZY Qu, DC Cong, Transactions of the Chinese Society for Agricultural Machinery, 49, 361-366 (2018) 